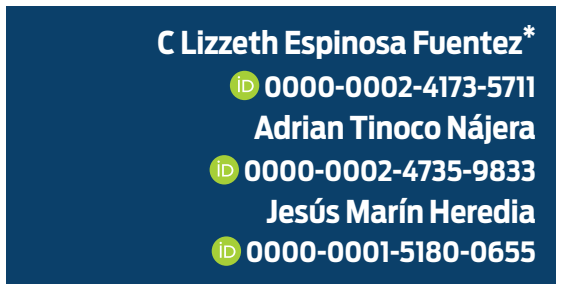

Hospital Veterinario de Especialidades, Departamento de Medicina Cirugía y Zootecnia de Pequeñas Especies, Facultad de Medicina Veterinaria y Zootecnia, Universidad Nacional Autónoma de México. Ciudad de México, México.

* Autor para correspondencia: Tel: (55) 40972982 Correo electrónico: lizzeth.ef@gmail.com
Recibido: 2018-10-12 Aceptado: 2018-12-13 Publicado: 2019-03-29

Información y declaraciones adicionales en la página 8

(c) Derechos de autor: C Lizzeth Espinosa Fuentez et al. 2019

acceso abierto $\mathbf{0}$

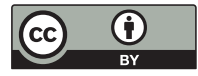

Distribuido bajo una Licencia Creative Commons Atribución 4.0 Internacional (CC-BY 4.0)

\section{Diagnóstico y tratamiento de un gato (Felis silvestris catus) con hiperaldosteronismo primario}

\section{Resumen}

Descripción del caso. Se presentó a consulta en el Hospital Veterinario de Especialidades (HVE) de la UNAM un paciente felino de 12 años con semiología compatible con miopatía hipocalémica con tres meses de evolución. En el examen físico general se observó condición corporal 2/5, posición plantígrada y ventroflexión cervical. Acudió a consulta con hemograma y bioquímica con valores alterados. Al realizar ultrasonido (US) abdominal, se evidenció proceso neoplásico en glándula adrenal.

Hallazgos clínicos e interpretación. Neutrofilia y linfopenia asociadas a estrés, incremento de AST y CK, e hipocalemia, asociados a miopatía hipocalémica, que en conjunto con las alteraciones en el ultrasonido fueron compatibles con hiperaldosteronismo primario, secundario a carcinoma corticoadrenal.

Tratamiento y evolución. Se descartaron otras afecciones que generan miopatía hipocalémica y se instauró tratamiento médico con espironolactona y gluconato de potasio, mejorando así su semiología.

Pruebas de laboratorio. Como las alteraciones en el hemograma y la bioquímica realizadas fuera del HVE eran compatibles con miopatía hipocalémica, se decartó enfermedad renal crónica debido a que los niveles de creatinina se encontraban dentro de los valores de referencia del laboratorio. La T4T estaba dentro de los intervalos de referencia. La aldosterona fue compatible con hiperaldosteronemia.

Discusión y relevancia clínica. El hiperaldosteronismo primario felino parece ser una enfermedad subdiagnosticada, porque los principales diagnósticos diferenciales para este padecimiento son el hipertiroidismo y la enfermedad renal crónica. Asimismo, estos pacientes pueden presentar hipertensión arterial sistémica y, hoy en día, se reconoce que el hiperaldosteronismo es la causa más común de hipertensión endocrina en gatos. Por lo tanto, es

\section{Una forma de citar este artículo:}

Espinosa-Fuentez CL, Tinoco-Nájera A, Marín Heredia J. Diagnóstico y tratamiento de un gato (Felis silvestris catus) con hiperaldosteronismo primario. Clínica Veterinaria: abordaje diagnóstico y terapéutico. 2019;5:e35201953. DOI: 10.22201/fmvz.23958766e.201935 
importante proporcionar bases y orientación a los médicos veterinarios para diagnosticar y tratar este padecimiento, debido a que en México no se cuenta con la prueba diagnóstica definitiva.

Palabras clave: hiperaldosteronismo primario felino, hipertensión, hiperaldosteronemia, hiperplasia adrenocortical, adenoma o carcinoma adrenocortical, miopatía hipocalémica.

\section{Diagnosis and treatment of a cat (Felis silvestris catus) with primary hyperaldosteronism}

\section{Abstract}

Case description. A 12 years old feline patient presented to the Hospital Veterinario de Especialidades (HVE) de la UNAM with clinical signs consistent with hypokalemic myopathy of three months duration. The physical exam revealed a body condition score of $2 / 5$, plantigrade stance and cervical ventroflexion. Previous complete blood count and biochemistry revealed abnormal values. Abdominal ultrasound was performed and identified neoplasia in the adrenal gland.

Diagnostics and interpretation. A stress leukogram characterized by neutrophilia and lymphopenia, increase in AST and CK, and hypokalemia associated with hypokalemic myopathy. These results along with ultrasonographic findings, were consistent with primary hyperaldosteronism secondary to adrenal carcinoma.

Treatment and clinical response. Other causes of hypokalemia and hypokalemic myopathy were ruled out. Treatment was initiated with oral spironolactone and potassium gluconate, with marked improvement of its clinical signs.

Laboratory tests. Based on the results of complete blood count and biochemistry performed outside the HVE consistent with hypokalemic myopathy, other etiologies such as renal disease was ruled out as the levels of creatinine were within the reference interval. The T4T was also within reference intervals. Aldosterone levels were consistent with hyperaldosteronemia.

Discussion and clinical relevance. Feline primary hyperaldosteronism appears to be an underdiagnosed disease, as diagnostic tests are limited to ruling out hyperthyroidism and chronic kidney disease. Similarly, these patients are likely to present with systemic hypertension and nowadays it is recognized that hyperaldosteronism is the most common cause of endocrine hypertension in cats. Therefore, it is important to educate and guide veterinarians in the diagnosis and treatment of this condition, as a final diagnostic test is not available in Mexico.

Keywords: Feline primary hyperaldosteronism, hypertension, hyperaldosteronemia, adreno-cortical hyperplasia, adrenal adenoma or carcinoma, hypokalemic myopathy. 


\section{Descripción del caso}

Acude a consulta un gato europeo doméstico, macho castrado de 12 años con calendario de vacunación y desparasitación vigente, mascota única, habita dentro de casa. Posee prueba de leucemia viral felina y SIDA felino negativa. El motivo de la consulta fue debilidad generalizada, ventroflexión cervical intermitente, hiporexia, posición plantígrada, disminución en la condición corporal con evolución de tres meses.

\section{Hallazgos clínicos e interpretación}

Un médico veterinario externo a este caso realizó hemograma y bioquímica en los que se reportan las siguientes alteraciones: leucograma de estrés por neutrofilia $17 \times 10^{9} / \mathrm{L}(2.5-12.5)$ y linfopenia $1.2 \times 10^{9} / \mathrm{L}$ (1.5-7). Incremento de AST $415 \mathrm{U} / \mathrm{L}(<61)$ y CK $9465 \mathrm{U} / \mathrm{L}(<277)$ asociado a daño muscular, hipocalemia $2.6 \mathrm{mmol} / \mathrm{L}$ (3.6-5.3) asociado a endocrinopatía sin descartar falta en el aporte. Debido a las alteraciones en las pruebas de sangre se inició el abordaje diagnóstico para afecciones que cursan con hipocalemia, por lo tanto se midió la T4T, que se encontró dentro de los intervalos de referencia. Los niveles de creatinina también estaban dentro de los valores de referencia $147 \mu \mathrm{mol} / \mathrm{L}$ (56-176). También se realizó ultrasonido (US) abdominal (Imagen 1), en el que se observó la glándula adrenal izquierda con patrón heterogéneo de $3.43 \times 2.91 \mathrm{~cm}$ adyacente al polo craneal del riñón izquierdo asociado a un proceso neoplásico. Tomando en cuenta las alteraciones en el ultrasonido, se sospechó de hiperaldosteronismo primario, por lo que se tomó una muestra para medir la aldosterona. Se midió la presión arterial sistémica, su valor sistólico fue de $143 \mathrm{mmHg}$, entonces al paciente se le consideró como prehipertenso (140-159 mmHG) de acuerdo con los valores de la IRIS ${ }^{1}$ (Sociedad Internacional de Interés Renal, por sus siglas en inglés). El día 21 de evolución se recibieron los resultados de la aldosterona: 4572 pmol/L (110-540) compatibles con hiperaldosteronemia.

\section{Tratamiento y evolución}

Después de tomar las muestras de sangre, se inició tratamiento mediante espironolactona a $4 \mathrm{mg} / \mathrm{kg}$ vía oral cada 12 h y gluconato de potasio a $4 \mathrm{mEq}$ totales vía oral cada 12 h, ambos hasta nueva indicación. En ese momento, no se midió el valor del potasio; sin embargo, estaba indicado medirlo y con base en el resultado determinar si era un paciente que debía ser hospitalizado.

El día 35 de evolución, los propietarios reportaron mejoría de los signos clínicos y se realizó tomografía computarizada (TC) de abdomen simple y contrastada, durante el procedimiento anestésico se puncionó el tumor y la muestra fue enviada a estudio histopatológico (Imagen 2). En la TC se observó una estructura hipodensa craneal al riñón izquierdo que por su localización corresponde a la glándula adrenal de $4.2 \times 3.7 \times 3.1 \mathrm{~cm}$, que invade la vena renal izquierda y la arteria aorta. Para el día 43 de evolución, el paciente fue sometido a adrenalectomía, ureterectomía y nefrectomía izquierdas. Los órganos fueron enviados a estudio histopatológico. 


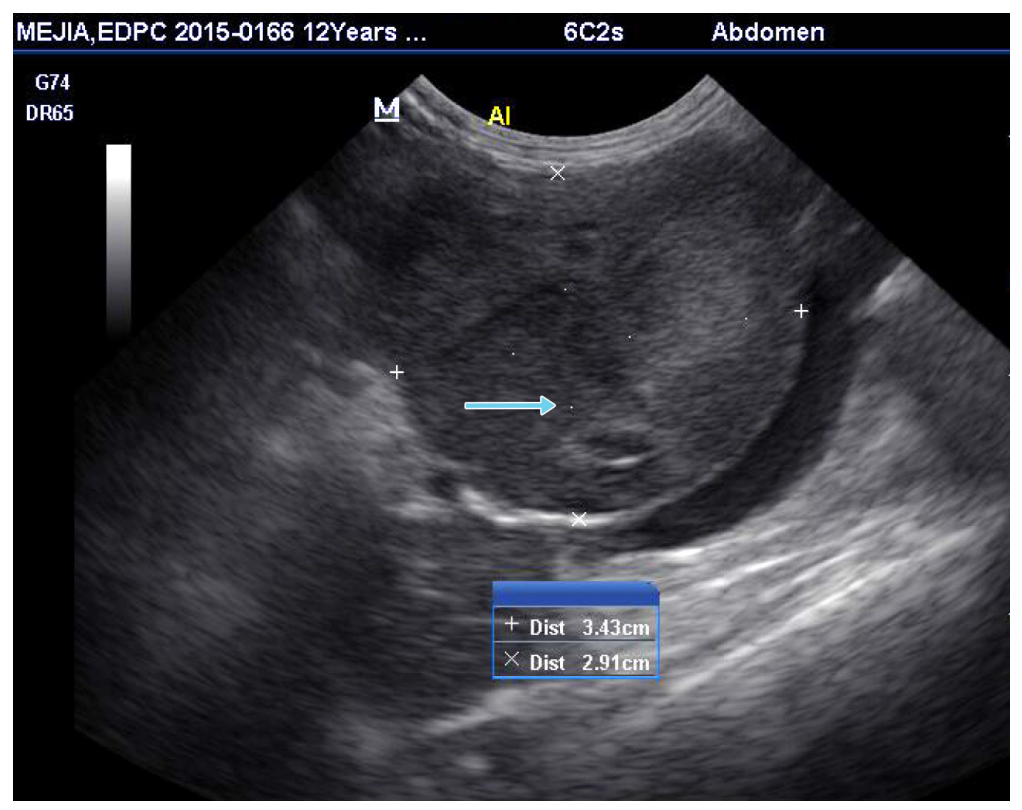

Imagen 1. Glándula adrenal izquierda (flecha azul) con patrón heterogéneo de $3.43 \times 2.91 \mathrm{~cm}$ adyacente al polo craneal del riñón izquierdo asociado a proceso neoplásico.

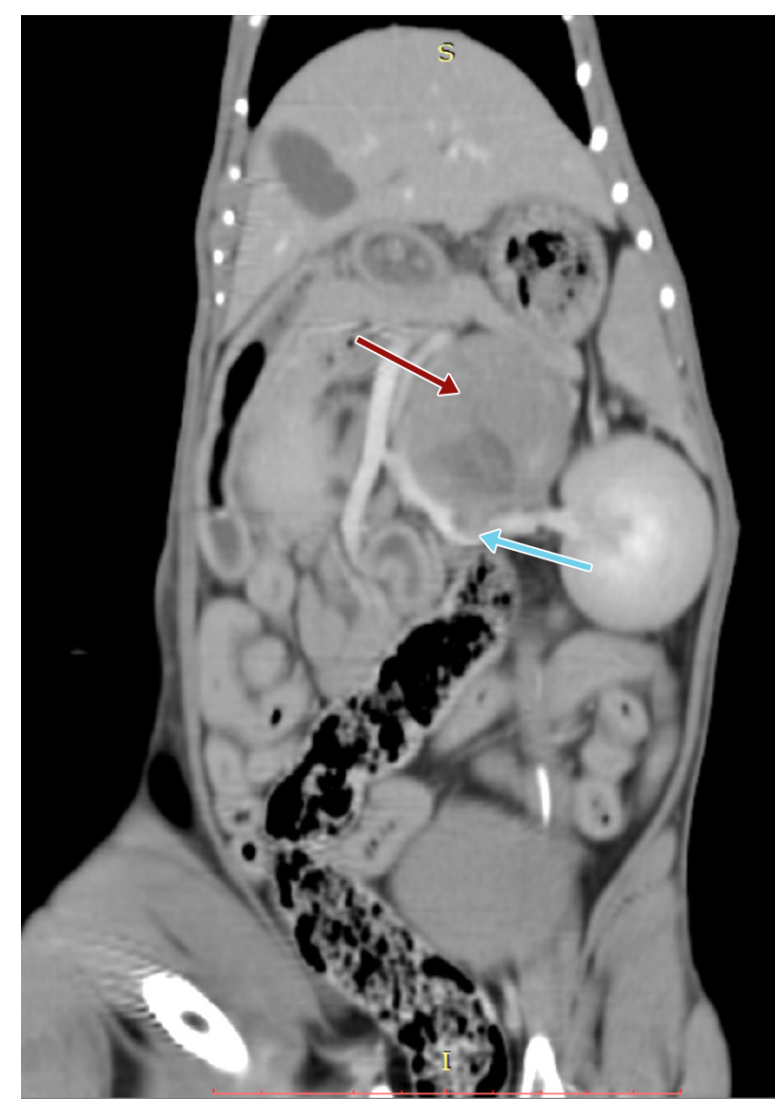

Imagen 2. Corte coronal de la TC de abdomen donde se observa una estructura hipodensa craneal al riñón izquierdo (flecha roja) que por su localización corresponde a la glándula adrenal de $4.2 \times 3.7 \times 3.1 \mathrm{~cm}$, que invade la vena renal izquierda (flecha azul) y la arteria aorta. 
El día 69 de evolución, el reporte histopatológico fue carcinoma corticoadrenal y glomerulonefritis membranoproliferativa. El paciente continuó con tratamiento durante dos meses a dosis reducción de espironolactona y gluconato de potasio. Se midieron los electrolitos control durante meses en los cuales no se encontraron alteraciones.

Catorce meses después del procedimiento quirúrgico el paciente acudió por el área de urgencias por presentar signos compatibles con miopatía hipocalémica. Se realizó gasometría, que reportaba pH 7.48 (7.35-7.46), pCO2 30.7 mmHg (36-44), HCO3 $22.7 \mathrm{mmol} / \mathrm{L}$ (18-24) presentando alcalemia por alcalosis respiratoria asociada a estrés o dolor. Hipocalemia de $1.7 \mathrm{mmol} / \mathrm{L}$ (3.5-5.5), hipocalcemia $0.72 \mathrm{mmol} / \mathrm{L}(1.20-1.50)$ e hipercloremia $123 \mathrm{mmol} / \mathrm{L}(107-117)$ asociadas a hiperaldosteronismo. Se hospitalizó al paciente con terapia de líquidos a terapia de mantenimiento con solución Hartmann e infusión de potasio a $0.5 \mathrm{mEq} / \mathrm{kg} / \mathrm{h}$ y espironolactona a $2 \mathrm{mg} / \mathrm{kg}$ vía oral cada 12 horas. Al día siguiente, se midió el control de electrolitos, aún persistía la hipocalemia de 2.1 mmol/L (3.5-5.5), también se realizó ultrasonido abdominal en el que se observaron lesiones hiperecóicas en hígado y retroperitoneo, sugerentes de metástasis tardía por implantación de células tumorales. Los propietarios optaron por la eutanasia y por asuntos personales no aceptaron que se realizara la necropsia.

\section{Discusión y relevancia clínica}

Los gatos diagnosticados con hiperaldosteronismo generalmente son gerontes, no parece haber predisposición por sexo o raza. ${ }^{2}$ Las principales alteraciones reportadas en la bioquímica son hipocalemia, hasta en el $87 \%$ de los pacientes, e incremento de CK al $93 \%$, secundaria al esfuerzo muscular del paciente por la debilidad muscular, ${ }^{2}$ ambas presentadas por el paciente del caso clínico. También se ha reportado hiperazotemia e incremento de AST. ${ }^{2}$ Las pruebas de sangre como primera herramienta son indispensables, ya que mediante ellas se descartan afecciones que cursan con miopatías hipocalémicas, tales como hipertiroidismo, diabetes y enfermedad renal crónica.

Estos pacientes suelen desarrollar polimiopatía hipocalémica, un trastorno muscular generalizado que resulta de los niveles séricos bajos de potasio ( $1.5 \mathrm{a}$ $3.5 \mathrm{mEq} / \mathrm{L}){ }^{3}$ lo que conduce a signos de debilidad, ventroflexión cervical, mialgia, marcha rígida y postura plantigrada de los miembros pélvicos. La principal complicación que desarrollan los pacientes hipocalémicos son arritmias ventriculares y refractariedad a algunos antiarrítmicos. ${ }^{3}$ La hipocalemia se puede corregir mediante infusión de potasio (de 0.1 a $0.5 \mathrm{mEq} / \mathrm{kg} / \mathrm{h}$ según el nivel de hipocalemia, el valor más alto se usa en pacientes con agotamiento grave de potasio) o mediante el uso de suplementos orales (citrato de potasio o gluconato: 1 a 4 mEq/gato PO BID). ${ }^{3}$

Con el paciente de este caso clínico, y el primer valor de potasio $(2.6 \mathrm{mmol} / \mathrm{L})$, lo indicado era su hospitalización con el fin de restablecer los valores de potasio. En este paciente se utilizó el valor más alto de infusión de potasio cuando acudió a urgencias, porque su hipocalemia era de $1.7 \mathrm{mmol} / \mathrm{L}$. La administración a tasas superiores a $0.5 \mathrm{mEq} / \mathrm{kg}$ también puede causar arritmias cardíacas graves o fatales. ${ }^{3}$

El hiperaldosteronismo es una endocrinopatía primaria o secundaria. Por lo general, la forma de descartar que sea secundaria es mediante estudios de imagen 
en los cuales se observe una o ambas glándulas adrenales afectadas. La primaria se diagnostica al medir la aldosterona en sangre, con un intervalo de referencia de 110 a $540 \mathrm{pmol} / \mathrm{L}$; 40 o $195 \mathrm{pg} / \mathrm{mL}^{4}$; relación renina:aldosterona en sangre (concentración plasmática de aldosterona: 110 a 540 pmol/L; 40 a 195 pg/mL y a una concentración plasmática de renina de 60 a $630 \mathrm{fmol} / \mathrm{L} / \mathrm{s} ; 0.3-3 \mathrm{ng} / \mathrm{mL} / \mathrm{h}$ ) con un intervalo de 0.3 a 3.84; o la relación aldosterona:creatinina en orina con un intervalo de referencia de $46.5\left(10^{9}\right) .^{5}$

La medición de aldosterona se incrementa hasta en el $82 \%$ de los casos, sin embargo, también hay pacientes dentro del intervalo, y eso se debe al efecto de la hipocalemia, ${ }^{2}$ por lo que, este hallazgo no descarta el padecimiento. La prueba diagnóstica de elección es la relación renina:aldosterona, sin embargo, aún no se encuentra disponible en México. En el paciente del presente caso clínico, la hiperaldosteronemia fue diagnóstica, pues como lo reporta la literatura, esta alteración se encuentra hasta en el $82 \%$ de los casos. ${ }^{2}$ Cuando el resultado se encuentra en el tercio alto de los valores de referencia, debe considerarse sospechoso. En ambos casos, deben considerarse estudios de imagen complementarios.

Este paciente, además de presentar carcinoma corticoadrenal, tenía glomerulonefritis membranoproliferativa, revelada por el estudio histopatológico. Se ha visto que la angiotensina II es un secretagogo de la aldosterona, un vasoconstrictor periférico y un regulador de la filtración glomerular, así como, un factor de crecimiento y una verdadera citoquina. ${ }^{6}$

Cada vez hay más pruebas de que la angiotensina II está implicada en la regulación de las respuestas inflamatorias y de las células inmunes y, por lo tanto, puede tener un papel activo en el reclutamiento de células inflamatorias en el riñón. La evidencia reciente indica que no solo la angiotensina ॥, sino también la aldosterona per se pueden contribuir a la progresión del daño renal al promover la trombosis y la fibrosis, ${ }^{6}$ por lo que el daño renal en este paciente pudo ser secundario al hiperaldosteronismo.

Así que, en este caso la enfermedad renal crónica, como causa de hipocalemia, se descartó debido a que los niveles de creatinina se encontraban dentro de los valores de referencia $147 \mu \mathrm{mol} / \mathrm{L}$, pero no se consideró la disminución de la condición corporal del paciente, por lo que el valor de creatinina pudo haber sido subestimado; por lo tanto, estaba indicado realizar prueba de SMDA (dimetilarginina simétrica) para confirmar o descartar enfermedad renal crónica, además, una vez confirmada la enfermedad renal crónica, se debía estadificar (medir la creatinina o SMDA) y subestadificar mediante la determinación de proteinuria (relación proteína:creatinina en orina) y presión arterial sistémica, con el fin de determinar el tratamiento adecuado de acuerdo con su estadio y con base en la estadificación de la IRIS.

La literatura reporta que las neoplasias funcionales de la zona glomerular no necesitan ser grandes para causar signos clínicos, en algunos casos las neoplasias pueden estar por debajo del límite de detección del US o incluso de la TC o la resonancia magnética $(\mathrm{RM}) .{ }^{7}$ Asimismo, la hiperplasia tampoco puede ser revelada por las técnicas convencionales de diagnóstico por imagen.

Existen reportes de neoplasias no funcionales que se detectan mediante imagen, pero pueden no causar signos clínicos. ${ }^{7}$ De manera que, un tumor suprarrenal visible puede no ser el causante de los signos. Si la cirugía se planifica solo a partir de imágenes de diagnóstico, se puede realizar una adrenalectomía incorrecta, ${ }^{7}$ es 
decir, del lado no afectado. En un estudio realizado por Kempers et al. (2009), concluyeron que la TC y RM no identifican con precisión la fuente de exceso de aldosterona en hasta el $38 \%, 8$ no obstante, si puede ser detectada hasta en el $62 \%$ de los casos como sucedió con el paciente descrito, así que, si bien el estudio de imagen forma parte del diagnóstico, no se considera una prueba única.

En el $57 \%$ de los pacientes con hiperaldosteronismo primario, la afección es consecuencia de carcinoma corticoadrenal, el $13 \%$ de adenoma y el $30 \%$ de hiperplasia nodular, ${ }^{7}$ además la literatura reporta que hasta el $78 \%$ es unilateral. Estos datos son de gran importancia ya que esta situación define el tratamiento a elegir.

En un estudio realizado por Ash et al. (2005), se revisaron 13 casos de los cuales seis eran consecuencia de carcinoma unilateral y siete de adenoma (cinco unilaterales y dos bilaterales); cabe destacar que, en todos ellos se evidencia hiperaldosteronemia, ${ }^{9}$ estos hallazgos demuestran que, en la mayoría de los pacientes, la afección será unilateral y mostrarán hiperaldosteronemia, tal como sucedió en el caso del paciente aquí citado.

La elección del tratamiento dependerá de los hallazgos en las pruebas antes descritas. Para gatos con enfermedad unilateral, la adrenalectomía sigue siendo el tratamiento de elección, parece ser curativa tanto para adenomas como para carcinomas y los signos resuelven sin tratamiento adicional, la cirugía normaliza el eje renina-angiotensina-aldosterona, resolviendo así la hipocalemia.

Tratamos medicamente al paciente con espironolactona y gluconato de potasio durante dos meses posteriores a la cirugía, después ya no fue necesario, debido a que no presentaba desbalances electrolíticos. En pacientes donde la afección es bilateral o se ha demostrado metástasis o invasión de la vena cava, el tratamiento es médico mediante espironolactona y antihipertensivos. Se ha visto que la hipertensión sistémica mejora y se cura en hasta en el $82 \%{ }^{4}$ Este paciente nunca presentó hipertensión sistémica. No obstante, en pacientes con hipertensión sistémica, uno de los principales motivos de consulta puede ser la pérdida de visión secundaria a desprendimiento de retina, la cual se ha asociado más en pacientes con hiperplasia suprarrenal bilateral. ${ }^{10}$

Para planear adecuadamente la cirugía es necesario realizar estudios de imagen. En pacientes con neoplasias suprarrenales, el US y la TC poseen especificidad del 80 y $100 \%$ respectivamente, y sensibilidad del 90 y $92 \%$ para la detección de invasión vascular. ${ }^{11}$ En el caso clínico aquí expuesto, la invasión se detectó mediante TC que posee mayor sensibilidad y especificidad, por lo que, sería el método de elección para poder planear la cirugía una vez establecido el diagnóstico.

Un estudio realizado por Lo et al. (2014) evaluó la adrenalectomía en diez pacientes con tumores unilaterales sin presencia aparente de metástasis. El estudio concluyó que la adrenalectomía es el tratamiento de elección, el tipo de tumor y la ubicación no afectaron el tiempo de sobrevida y los signos resolvían por completo ${ }^{12}$; sí se reportó que complicaciones, tales como hemorragias, se presentaban en el periodo postquirúrgico inmediato; el único factor significativo que afectó el tiempo medio de supervivencia fue el tiempo de anestesia mayor a cuatro horas.

El pronóstico para pacientes con neoplasia unilateral después de la cirugía, si sobreviven al periodo postoperatorio inmediato, es excelente. Los pacientes sometidos a cirugía pueden alcanzar tiempos de sobrevida de hasta 3.5 a 5 años, pero 
en aquellos que solo fueron tratados medicamente, la sobrevida reportada es de hasta 984 días. $^{10}$

La muerte se asocia normalmente con enfermedad renal crónica progresiva o hipertensión refractaria. El pronóstico a largo plazo de los gatos con hiperaldosteronismo primario, secundario a hiperplasia bilateral de la zona glomerulosa no está bien documentado. El paciente de este caso clínico fue eutanasiado después de documentarse la metástasis, sin embargo, había sobrevivido 14 meses después de la cirugía sin presentar signos clínicos, así, la sobrevida puede variar dependiendo de la presencia de metástasis; pero, en la literatura no está reportado un tiempo medio de sobrevida para estos pacientes.

El hiperaldosteronismo aunque aún se considera una condición poco común, se debe tomar en cuenta en gatos de mediana a mayor edad con miopatía hipocalémica e hipertensión sistémica refractaria al tratamiento. Parece ser un padecimiento subdiagnosticado debido a que la medición de la presión arterial no forma parte de la evaluación rutinaria de los pacientes y en aquellos con hipocalemia, ésta solo se trata al igual que la hipertensión sin indagar acerca del origen. Para poder establecer el diagnóstico es importante tener en cuenta los hallazgos en los estudios de sangre, en todos aquellos pacientes con hipocalemia está justificado realizar US abdominal en busca de irregularidades en las adrenales. Una vez establecido el diagnóstico la adrenalectomía siempre será la primera opción terapéutica en pacientes con afección unilateral, aunque debe planearse mediante TC para determinar si hay compromiso o posibles metástasis, o invasión a órganos adyacentes como grandes vasos. En pacientes con hiperplasia de la zona glomerulosa bilateral, neoplasia unilateral no resecable o metástasis, es posible el tratamiento médico y los tiempos de sobrevida varían de acuerdo al tratamiento establecido, que deberá ser planeado para cada paciente de acuerdo con los hallazgos.

\section{Financiamiento}

Pruebas de imagen, histopatología y patología clínica financiadas por el propietario.

\section{Conflictos de interés}

Los autores de este trabajo declaran no tener conflicto de intereses.

\section{Contribución de los autores}

CLEF redató el manuscrito y aprobó la versión final antes de publicarse.

ATN dio seguimiento a la evaluación del paciente, tomó las decisiones clínicas y aprobó la versión final del manuscrito antes de publicarse.

JMH hizo la revisión crítica del manuscrito y aprobó la versión final antes de publicarse.

\section{Referencias}

1. International Renal Interest Society (IRIS). IRIS staging of CKD (modified 2017). Disponible en: http://www.iris-kidney.com/pdf/IRIS_2017_Staging_of_CKD_ 09May 18.pdf

2. Schulman RL. Feline primary hyperaldosteronism. Veterinary Clinics: Small Animal Practice. 2010;40(2):353-9. 
3. Little SE. The Cat. Clinical medicine and management. London (EN): Elsevier, Saunders, 2012.

4. Javadi S, Slingerland LI, Van de Beek MG, Boer P, Boer WH, Mol JA, Rijberk A, Kooistra HS. Plasma renin activity and plasma concentrations of aldosterone, cortisol, adrenocorticotropic hormone, and $\alpha$-melanocyte-stimulating hormone in healthy cats. Journal of Veterinary Internal Medicine. 2004;18(5):625-31.

5. Koutinas CK, Soubasis NC, Djajadiningrat-Laanen SC, Kolia E, Theodorou K. Urinary aldosterone/creatinine ratio after fludrocortisone suppression consistent with PHA in a cat. Journal of the American Animal Hospital Association. 2015;51(5):338-41.

6. Javadi S, Djajadiningrat-Laanen SC, Kooistra HS, Van Dongen AM, Voorhout G, Van Sluijs FJ, Van Den TSGAM, Rijnberk A. Primary hyperaldosteronism, a mediator of progressive renal disease in cats. Domestic Animal Endocrinology. 2005;28(1):85-104.

7. Djajadiningrat-Laanen SC, Galac S, Kooistra H. Primary hyperaldosteronism: expanding the diagnostic net. Journal of Feline Medicine and Surgery. $2011 ; 13(9): 641-50$.

8. Kempers M, Lenders J, Van Outheusden L. Systematic review: diagnostic procedures to differentiate unilateral from bilateral adrenal abnormality in primary aldosteronism. Ann Intern Med. 2009;151:329-37.

9. Ash RA, Harvey AM, Tasker S. Primary hyperaldosteronism in the cat: a series of 13 cases. Journal of Feline Medicine \& Surgery. 2005;7(3):173-82.

10. Fernandez Y, Murgia D. Feline primary hyperaldosteronism: clinical and surgical approach. Companion Animal. 2016;21(3):146-53.

11. Mitchell JW, Mayhew PD, Culp WT, Brad Case J, Singh A, Fuller MC, Della Maggiore A. Outcome of laparoscopic adrenalectomy for resection of unilateral noninvasive adrenocortical tumors in 11 cats. Veterinary Surgery. 2017;46(5):714-21.

12. Lo AJ, Holt DE, Brown DC, Schlicksup MD, Orsher RJ, Agnello KA. Treatment of aldosterone-secreting adrenocortical tumors in cats by unilateral adrenalectomy: 10 cases (2002-2012). Journal of Veterinary Internal Medicine. 2014;28(1):137-43. 